\title{
Random eigenvalue problems revisited
}

\author{
S ADHIKARI \\ Department of Aerospace Engineering, University of Bristol, Queens Building, \\ University Walk, Bristol BS8 1TR, UK \\ e-mail: S.Adhikari@bristol.ac.uk
}

\begin{abstract}
The description of real-life engineering structural systems is associated with some amount of uncertainty in specifying material properties, geometric parameters, boundary conditions and applied loads. In the context of structural dynamics it is necessary to consider random eigenvalue problems in order to account for these uncertainties. Within the engineering literature, current methods to deal with such problems are dominated by approximate perturbation methods. Some exact methods to obtain joint distribution of the natural frequencies are reviewed and their applicability in the context of real-life engineering problems is discussed. A new approach based on an asymptotic approximation of multi-dimensional integrals is proposed. A closed-form expression for general order joint moments of arbitrary numbers of natural frequencies of linear stochastic systems is derived. The proposed method does not employ the 'small randomness' assumption usually used in perturbation based methods. Joint distributions of the natural frequencies are investigated using numerical examples and the results are compared with Monte Carlo simulation.
\end{abstract}

Keywords. Random eigenvalue problem; quadratic forms; asymptotic analysis; statistical distributions; linear stochastic systems.

\section{Introduction}

Characterization of natural frequencies and modeshapes play a fundamental role in the analysis and design of engineering dynamic systems. The determination of natural frequency and mode shapes requires the solution of eigenvalue problems. These problems also arise in the context of the stability analysis of structures. An eigenvalue problem could either be a differential eigenvalue problem or a matrix eigenvalue problem, depending on whether a continuous model or a discrete model is used to describe the given vibrating system. Descriptions of reallife engineering structural systems are inevitably associated with some amount of uncertainty in specifying material properties, geometric parameters, boundary conditions and applied loads. When we take account of these uncertainties, it is necessary to consider random eigenvalue problems. Several studies have been conducted on this topic since the mid-sixties. The

A list of symbols is given at the end of the paper

This paper is dedicated to Prof R N Iyengar of the Indian Institute of Science on the occasion of his formal retirement. 
study of probabilistic characterization of the eigensolutions of random matrix and differential operators is now an important research topic in the field of stochastic structural mechanics. The paper by Boyce (1968) and the book by Scheidt \& Purkert (1983) are useful sources of information on the early work in this area of research and also provide a systematic account of different approaches to random eigenvalue problems. Several review papers, for example, by Ibrahim (1987), Benaroya (1992), Manohar \& Ibrahim (1999) and Manohar \& Gupta (2003) have appeared in this field, and summarize current as well as earlier efforts.

In one of the earliest works on distributed systems, Iyengar \& Manohar (1989) have obtained the probability distribution function of the eigenvalues of a string with random properties. In this paper, we obtain a closed-form expression of arbitrary order joint moments of the natural frequencies of discrete linear systems or discretized continuous systems. The random eigenvalue problem of undamped or proportionally damped systems can be expressed by

$$
\mathbf{K}(\mathbf{x}) \phi_{j}=\omega_{j}^{2} \mathbf{M}(\mathbf{x}) \phi_{j} .
$$

Here $\omega_{j}$ and $\phi_{j}$ are the natural frequencies and mode shapes of the dynamic system. $\mathbf{M}(\mathbf{x})$ : $\mathbb{R}^{m} \mapsto \mathbb{R}^{N \times N}$ and $\mathbf{K}(\mathbf{x}): \mathbb{R}^{m} \mapsto \mathbb{R}^{N \times N}$, the mass and stiffness matrices, are assumed to be smooth, continuous and at least twice-differentiable functions of a random parameter vector $\mathbf{x} \in \mathbb{R}^{m}$. The vector $\mathbf{x}$ may consist of material properties, e.g., mass density, Poisson's ratio, Young's modulus or; geometric properties, e.g., length, thickness, and boundary conditions. Statistical properties of the system are completely described by the joint probability density function $p_{\mathbf{x}}(\mathbf{x}): \mathbb{R}^{m} \mapsto \mathbb{R}$. For mathematical convenience we express

$$
p_{\mathbf{x}}(\mathbf{x})=\exp \{-L(\mathbf{x})\},
$$

where $-L(\mathbf{x})$ is often known as the log-likelihood function. For example, if $\mathbf{x}$ is a $m$ dimensional multivariate Gaussian random vector with mean $\boldsymbol{\mu} \in \mathbb{R}^{m}$ and covariance matrix $\boldsymbol{\Sigma} \in \mathbb{R}^{m \times m}$ then

$$
L(\mathbf{x})=\frac{m}{2} \ln (2 \pi)+\frac{1}{2} \ln \|\boldsymbol{\Sigma}\|+\frac{1}{2}(\mathbf{x}-\boldsymbol{\mu})^{T} \boldsymbol{\Sigma}^{-1}(\mathbf{x}-\boldsymbol{\mu}) .
$$

It is assumed that in general the random parameters are non-Gaussian and correlated, i.e., $L(\mathbf{x})$ can have any general form provided it is a smooth, continuous and at least twice-differentiable function. It is further assumed that $\mathbf{M}$ and $\mathbf{K}$ are symmetric and positive definite random matrices so that all the eigenvalues are real and positive.

The aim of studying random eigenvalue problems is to obtain the joint probability density function (pdf) of the eigenvalues and the eigenvectors. The current literature on random eigenvalue problems arising in engineering systems is dominated by perturbation methods. These methods work well when the uncertainties are small and the parameter distribution is Gaussian. Methods which are not based on the perturbation approach but still have the generality and computational efficiency to be applicable for engineering dynamic systems are rare. Grigoriu (1992) has examined the roots of characteristic polynomials of real symmetric random matrices using distributions of zeros of random polynomials. Lee \& Singh (1994) have proposed a direct matrix product (Kronecker product) method to obtain the first two moments of the eigenvalues of discrete linear systems. Nair \& Keane (2003) have proposed a stochastic reduced basis approximation which can be applied to discrete or discretized continuous dynamic systems. Recently Adhikari \& Langley (2003) and Adhikari \& Friswell (2004) have proposed some non-perturbative methods to obtain moments and pdf of the eigenvalues for the general case. 
For dynamic response analysis and in many other practical problems, joint distributions of the eigenvalues are required. Under very special circumstances, for example when the system matrix is GUE (Gaussian unitary ensemble) or GOE (Gaussian orthogonal ensemble), an exact closed-form expression can be obtained for the joint pdf of the eigenvalues (Mehta 1991). However, unfortunately, the system matrices of real structures may not always follow such distributions, and consequently some kind of approximate analysis is required for structural engineering problems. While several papers are available on the distribution of individual eigenvalues, joint distributions of the eigenvalues seem to have received little attention in the literature. To the best of the authors knowledge only first-order perturbation results (Collins \& Thomson 1969) are available for the joint pdf of the eigenvalues. In this paper, a few methods are proposed to obtain joint distributions of the natural frequencies of discrete linear systems. In $\S 2$, some exact results available for the joint probability density function of the eigenvalues are presented and their limitations in the context of engineering dynamic systems have been discussed. In $\S 3$, first- and second-order perturbation methods are discussed and the joint statistics of the natural frequencies are derived for the special case when the basic random variables are Gaussian. When the basic random variables are non-Gaussian, a new method to obtain arbitrary order moments of the natural frequencies is proposed in $\S 4$. In $\S 5$, the proposed analytical methods are applied to a three degree-of-freedom system and the results are compared with Monte Carlo simulations.

\section{Exact joint probability density function of the natural frequencies}

Without any loss of generality, the eigenvalue problem in (1) can be expressed by

$$
\mathbf{H}(\mathbf{x}) \boldsymbol{\psi}_{j}=\omega_{j}^{2} \boldsymbol{\psi}_{j},
$$

where

$$
\begin{aligned}
& \mathbf{H}(\mathbf{x})=\mathbf{M}^{-1 / 2}(\mathbf{x}) \mathbf{K}(\mathbf{x}) \mathbf{M}^{-1 / 2}(\mathbf{x}) \in \mathbb{R}^{N \times N} \\
& \text { and } \quad \boldsymbol{\psi}_{j}=\mathbf{M}^{1 / 2} \phi_{j} .
\end{aligned}
$$

Since $\mathbf{M}$ and $\mathbf{K}$ are assumed to be real, symmetric and positive definite, the matrix $\mathbf{H}$ (the dependence on $\mathbf{x}$ will be omitted for notational convenience) given in (5) is also a real, symmetric and positive definite matrix. Therefore, its eigenvalues are positive and the matrix of eigenvectors,

$$
\boldsymbol{\Psi}=\left[\boldsymbol{\psi}_{1}, \boldsymbol{\psi}_{2}, \ldots, \boldsymbol{\psi}_{N}\right] \in \mathbb{R}^{N \times N},
$$

can be normalized into an orthogonal matrix. The matrix $\Psi$ therefore belongs to the group of $N \times N$ orthogonal matrices, denoted by $O(N)$. For convenience, we arrange the natural frequencies in ascending order and construct a diagonal matrix containing the natural frequencies as

$$
\Omega=\operatorname{diag}\left[\omega_{1}, \omega_{2}, \ldots, \omega_{N}\right] \quad \text { where } \quad 0<\omega_{1}<\omega_{2} \cdots<\omega_{N} .
$$

Using $\Psi$, the system matrix can be transformed to $\Omega$ as

$$
\mathbf{H}=\Psi \Omega^{2} \Psi^{T} .
$$


From the probability density function of $\mathbf{x}$ it is possible to obtain the probability density function of $\mathbf{H}$, denoted by $p_{\mathbf{H}}(\mathbf{H})$. Since it is assumed that the natural frequencies are distinct, the probability of occurrence of $\omega_{i}=\omega_{j}$, for $i \neq j$, is zero. Under these fairly general and physically relevant assumptions, the joint pdf of the natural frequencies can be obtained using Muirhead (1982, theorem 3.2.17). The exact joint probability density function of all the $N$ natural frequencies of the system is described in the following theorem.

Theorem 1. The joint probability density function of the natural frequencies of an $\mathrm{N}$ dimensional linear positive definite dynamic system is given by,

$$
\begin{aligned}
& p_{\Omega}\left(\omega_{1}, \omega_{2}, \ldots, \omega_{N}\right) \\
& \quad=\frac{\pi^{N^{2} / 2}}{\Gamma(N / 2)} \prod_{i<j \leq N}\left(\omega_{j}^{2}-\omega_{i}^{2}\right) \int_{O(N)} p_{\mathbf{H}}\left(\Psi \Omega^{2} \Psi^{T}\right)(d \Psi),
\end{aligned}
$$

where $\mathbf{H}=\mathbf{M}^{-1 / 2} \mathbf{K M}^{-1 / 2}$ and $p_{\mathbf{H}}(\mathbf{H})$ is the $p d f$ of $\mathbf{H}$.

By considering the Jacobian of the transformation in (9), the theorem can be proved following the procedure described by Muirhead (1982). In the product term in (10), $i$ varies from 1 to $N$ and for each value of $i, j$ varies from $i+1$ to $N$ so that there are a total of $N(N-1) / 2$ terms. The integral in (10) needs to be evaluated over the space of $N \times N$ orthogonal matrices. The probability density function of the system if the matrix $\mathbf{H}$ in theorem 1 can be any general smooth continuous density function and it is not restricted to the Gaussian density function. Although theorem 1 gives the exact closed-form joint pdf of all the natural frequencies of a linear stochastic system with general non-Gaussian parameter distribution, the following difficulties may be encountered for real-life engineering systems.

- The multi-dimensional integral over the orthogonal group $O(N)$ is difficult to carry out in practice and exact closed-form results can be derived only for few special cases;

- the derivation of an expression of the joint pdf of the system matrix $p_{\mathbf{H}}(\mathbf{H})$ is non-trivial even if the joint pdf of the random system parameters $\mathbf{x}$ is known;

- even if we can overcome the previous two problems, the joint pdf of the natural frequencies given by (10) is 'too much of information' to be useful for practical problems because

- it is not easy to 'visualize' the joint pdf in the space of $N$ natural frequencies, and

- the derivation of the marginal density functions of the natural frequencies from (10) is not straightforward, especially when $N$ is large.

In spite of these difficulties, an expression of joint pdf of the natural frequencies can be useful in many situations, for example, to verify the accuracy of an approximate numerical method. Here we list some special cases for which the integral in (10) can be carried out analytically and simple closed-form expressions can be obtained for the joint pdf of the natural frequencies.

- System matrix $\mathbf{H}$ is from a Gaussian orthogonal ensemble (GOE): This case forms the basis of random matrix theory (RMT) (Mehta 1991) and has been used extensively in the literature of different subjects, for example, in the study of excitation spectra of various atomic nuclei (Brody et al 1981) and high frequency structural vibrations (Langley \& Brown 2004a,b). With GOE assumption, the ensemble is invariant under every orthogonal transformation,

$$
\mathbf{H} \longrightarrow \boldsymbol{\Psi}^{T} \mathbf{H} \boldsymbol{\Psi},
$$


and various elements of $\mathbf{H}_{i j}, i \leq j$ are statistically independent and Gaussian. The pdf of $\mathbf{H}$ can be expressed as,

$$
p_{\mathbf{H}}(\mathbf{H})=\exp \left(-\theta_{2} \operatorname{Trace}\left(\mathbf{H}^{2}\right)+\theta_{1} \operatorname{Trace}(\mathbf{H})+\theta_{0}\right),
$$

where $\theta_{2}$ is real and positive, and $\theta_{1}$ and $\theta_{0}$ are real. If these two assumptions are satisfied, the joint pdf of the natural frequencies can be obtained (see Mehta 1991, chapter 3) as,

$$
p_{\Omega}\left(\omega_{1}, \omega_{2}, \ldots, \omega_{N}\right)=\exp \left[-\left(\sum_{j=1}^{N} \theta_{2} \omega_{j}^{4}-\theta_{1} \omega_{j}^{2}-\theta_{0}\right)\right] \prod_{i<j}\left|\omega_{j}^{2}-\omega_{i}^{2}\right| .
$$

Although the system matrices of a real-life structural system may not exactly follow the assumption of GOE, the distribution of the spacing between the natural frequencies is very close to that of a GOE matrix (Langley \& Brown 2001).

- System matrix $\mathbf{H}$ has Wishart distribution: If $\mathbf{Z} \in \mathbb{R}^{N \times N}$ is a Gaussian random matrix with zero mean and covariance matrix $\left[\mathbf{I}_{N} \otimes \mathbf{\Sigma}\right] \in \mathbb{R}^{N^{2} \times N^{2}}$, then the matrix $\mathbf{H}=\mathbf{Z}^{T} \mathbf{Z}$ is said to have a Wishart distribution (see, for example, Gupta \& Nagar 2000), commonly denoted as, $W_{N}(N, \Sigma)$. The pdf of $\mathbf{H}$ can be expressed by

$$
p_{\mathbf{H}}(\mathbf{H})=\frac{1}{2^{N^{2} / 2} \Gamma_{N}(N / 2)\|\boldsymbol{\Sigma}\|^{N} / 2} \operatorname{etr}\left(-\frac{1}{2} \boldsymbol{\Sigma}^{-1} \mathbf{H}\right)\|\mathbf{H}\|^{-1 / 2} .
$$

The spectrum of $\mathbf{H}$ in (5) is exactly the same as that of $\mathbf{M}^{-1} \mathbf{K}$. Therefore, if the elements of $\mathbf{M}^{-1}$ and $\mathbf{K}$ are uncorrelated and have Gaussian distribution, then $\mathbf{H}=\mathbf{M}^{-1} \mathbf{K}$ has Wishart distribution. If $\mathbf{H}$ has a distribution $W_{N}\left(N, \lambda \mathbf{I}_{N}\right)$, then the joint pdf of the natural frequencies can be expressed (see Muirhead 1982, theorem 3.2.19) as

$$
\begin{aligned}
& p_{\Omega}\left(\omega_{1}, \omega_{2}, \ldots, \omega_{N}\right) \\
& \quad=\frac{\pi^{N^{2} / 2}}{(2 \lambda)^{N^{2} / 2}\left(\Gamma_{N}(N / 2)\right)^{2}} \exp \left(-\frac{1}{2 \lambda} \sum_{i=1}^{N} \omega_{i}^{2}\right) \prod_{i=1}^{N} \frac{1}{\omega_{i}} \prod_{i<j}^{N}\left(\omega_{j}^{2}-\omega_{i}^{2}\right) .
\end{aligned}
$$

Some other exact expressions of the joint probability density function of the eigenvalues of a matrix following more general distributions can be found in the books by Mehta (1991) and Muirhead (1982), and in the paper by Dumitriu \& Edelman (2002). Unfortunately, however, the system matrices of real structures may not always follow such distributions and consequently some kind of approximate analysis is required. The rest of this paper is aimed at developing approximate methods to obtain joint statistics of the natural frequencies.

\section{Statistics of the natural frequencies using perturbation methods}

Using the first-order perturbation method, Collins \& Thomson (1969) derived the joint probability density function of the eigenvalues and eigenvectors of linear systems with uncertain parameters following Gaussian distribution. Over the past three decades, this work has inspired many authors to apply the first-order perturbation method in various problems of practical interest, see for example Hasselman \& Hart 1972, Hart 1973, Ramu \& Ganesan 1993 and Sankar et al 1993. Adhikari 2004 has used the first-order perturbation method in complex random eigenvalue problems arising in non-proportionally damped systems. The 
widespread application of the first-order perturbation method is primarily due to its computational efficiency as compared to other methods. The limitations of this method are well understood - if uncertainties in the system parameters are 'large' so that the 'spread' of the natural frequencies is close to their mean spacings then the method breaks down. This often happens in the case of higher natural frequencies. Moreover, if the elements of $\mathbf{x}$ are non-Gaussian then the first-order perturbation method is not helpful because there is no general method to obtain the resulting pdf in a simple manner. The accuracy and range of applicability of the first-order perturbation method can be extended if higher-order terms are used. Here, we briefly review the joint statistics of natural frequencies using the second-order perturbation method.

\subsection{Perturbation expansion}

The mass and the stiffness matrices are in general nonlinear functions of the random vector $\mathbf{x}$. Denote the mean of $\mathbf{x}$ as $\boldsymbol{\mu} \in \mathbb{R}^{m}$, and consider that

$$
\mathbf{M}(\boldsymbol{\mu})=\overline{\mathbf{M}}, \quad \text { and } \quad \mathbf{K}(\boldsymbol{\mu})=\overline{\mathbf{K}},
$$

are the 'deterministic parts' of the mass and stiffness matrices respectively. In general, $\overline{\mathbf{M}}$ and $\overline{\mathbf{K}}$ are different from the mean matrices. The deterministic part of the natural frequencies:

$$
\bar{\omega}_{j}=\omega_{j}(\boldsymbol{\mu})
$$

is obtained from the deterministic eigenvalue problem:

$$
\overline{\mathbf{K}} \bar{\phi}_{j}=\bar{\omega}_{j}^{2} \overline{\mathbf{M}} \bar{\phi}_{j}
$$

The natural frequencies, $\omega_{j}(\mathbf{x}): \mathbb{R}^{m} \mapsto \mathbb{R}$ are nonlinear functions of the parameter vector $\mathbf{x}$. If the natural frequencies are not repeated, then each $\omega_{j}(\mathbf{x})$ is expected to be a smooth and twice-differentiable function since the mass and stiffness matrices are smooth and twicedifferentiable functions of the random parameter vector. In the perturbation approach, the function $\omega_{j}(\mathbf{x})$ is expanded by its Taylor series about the point $\mathbf{x}=\boldsymbol{\mu}$ as

$$
\omega_{j}(\mathbf{x}) \approx \omega_{j}(\boldsymbol{\mu})+\mathbf{d}_{\omega_{j}}^{T}(\boldsymbol{\mu})(\mathbf{x}-\boldsymbol{\mu})+\frac{1}{2}(\mathbf{x}-\boldsymbol{\mu})^{T} \mathbf{D}_{\omega_{j}}(\boldsymbol{\mu})(\mathbf{x}-\boldsymbol{\mu}) .
$$

Here $\mathbf{d}_{\omega_{j}}(\boldsymbol{\mu}) \in \mathbb{R}^{m}$ and $\mathbf{D}_{\omega_{j}}(\boldsymbol{\mu}) \in \mathbb{R}^{m \times m}$ are respectively the gradient vector and the Hessian matrix of $\omega_{j}(\mathbf{x})$ evaluated at $\mathbf{x}=\boldsymbol{\mu}$, that is

$$
\begin{aligned}
\left\{\mathbf{d}_{\omega_{j}}(\boldsymbol{\mu})\right\}_{k} & =\left.\frac{\partial \omega_{j}(\mathbf{x})}{\partial x_{k}}\right|_{\mathbf{x}=\boldsymbol{\mu}} \\
\text { and }\left\{\mathbf{D}_{\omega_{j}}(\boldsymbol{\mu})\right\}_{k l} & =\left.\frac{\partial^{2} \omega_{j}(\mathbf{x})}{\partial x_{k} \partial x_{l}}\right|_{\mathbf{x}=\boldsymbol{\mu}}
\end{aligned}
$$

The expressions of the elements of the gradient vector and the Hessian matrix are given in appendix A. Due to (17), (19) implies that the natural frequencies are effectively expanded about their corresponding deterministic values $\bar{\omega}_{j}$.

Equation (19) represents a quadratic form in basic non-Gaussian random variables. The first-order perturbation expression can be obtained from (19) by neglecting the Hessian matrix. In this case the natural frequencies are simple linear functions of the basic random variables. This formulation is expected to produce acceptable results when the random variation in 
$\mathbf{x}$ is small and centred around the mean value. If the basic random variables are Gaussian then first-order perturbation results in a joint Gaussian distribution of the natural frequencies (Collins \& Thomson 1969). In this case a closed-form expression for their joint probability density function can be obtained easily. When the second-order terms are retained in (19), each $\omega_{j}(\mathbf{x})$ results in a quadratic form in $\mathbf{x}$. If the elements of $\mathbf{x}$ are Gaussian, it is possible to obtain joint statistics using the theory of quadratic forms as discussed in the next subsection.

\subsection{Joint statistics of the natural frequencies}

Discussions on quadratic forms in Gaussian random variables can be found in the books by (Johnson \& Kotz 1970, chap. 29) and Mathai \& Provost 1992. Using the methods outlined in these references, joint moments/cumulants of the natural frequencies are obtained in this section.

Considering $\mathbf{x}$ a multivariate Gaussian random vector with mean $\boldsymbol{\mu} \in \mathbb{R}^{m}$ and covariance matrix $\boldsymbol{\Sigma} \in \mathbb{R}^{m \times m}$, the joint moment generating function of $\omega_{j}(\mathbf{x})$ and $\omega_{k}(\mathbf{x})$, for any $s_{1}, s_{2} \in$ $\mathbb{C}$, can be obtained as

$$
\begin{aligned}
M_{\omega_{j}, \omega_{k}}\left(s_{1}, s_{2}\right)= & \mathrm{E}\left[\exp \left\{s_{1} \omega_{j}(\mathbf{x})+s_{2} \omega_{k}(\mathbf{x})\right\}\right] \\
= & \int_{\mathbb{R}^{m}} \exp \left\{s_{1} \omega_{j}(\mathbf{x})+s_{2} \omega_{k}(\mathbf{x})-L(\mathbf{x})\right\} \mathrm{d} \mathbf{x} \\
= & (2 \pi)^{-m / 2}\|\boldsymbol{\Sigma}\|^{-1 / 2} \\
& \times \int_{\mathbb{R}^{m}} \exp \left\{s_{1} \omega_{j}(\mathbf{x})+s_{2} \omega_{k}(\mathbf{x})-\frac{1}{2}(\mathbf{x}-\boldsymbol{\mu})^{T} \boldsymbol{\Sigma}^{-1}(\mathbf{x}-\boldsymbol{\mu})\right\} \mathrm{d} \mathbf{x} .
\end{aligned}
$$

Applying the quadratic expansion in (19) for $j$ and $k$, and rearranging the terms within the exponent, we have

$$
\begin{aligned}
& s_{1} \bar{\omega}_{j}+s_{2} \bar{\omega}_{k}+\left(s_{1} \mathbf{d}_{\omega_{j}}+s_{2} \mathbf{d}_{\omega_{k}}\right)^{T}(\mathbf{x}-\boldsymbol{\mu}) \\
& \quad-\frac{1}{2}(\mathbf{x}-\boldsymbol{\mu})^{T}\left[\boldsymbol{\Sigma}^{-1}-s_{1} \mathbf{D}_{\omega_{j}}-s_{2} \mathbf{D}_{\omega_{k}}\right](\mathbf{x}-\boldsymbol{\mu}) .
\end{aligned}
$$

The dependence on $\boldsymbol{\mu}$ has been omitted for notational convenience. Using the transformation $\mathbf{y}=(\mathbf{x}-\boldsymbol{\mu})$ the integral in (22) can be evaluated exactly

$$
\begin{aligned}
& M_{\omega_{j}, \omega_{k}}\left(s_{1}, s_{2}\right)=\left\|\mathbf{I}-s_{1} \mathbf{D}_{\omega_{j}} \boldsymbol{\Sigma}-s_{2} \mathbf{D}_{\omega_{k}} \boldsymbol{\Sigma}\right\|^{-1 / 2} \exp \left\{\left(s_{1} \bar{\omega}_{j}+s_{2} \bar{\omega}_{k}\right)\right. \\
& \left.+\frac{1}{2}\left(s_{1} \mathbf{d}_{\omega_{j}}+s_{2} \mathbf{d}_{\omega_{k}}\right)^{T} \boldsymbol{\Sigma}\left[\mathbf{I}-s_{1} \mathbf{D}_{\omega_{j}} \boldsymbol{\Sigma}-s_{2} \mathbf{D}_{\omega_{k}} \boldsymbol{\Sigma}\right]^{-1}\left(s_{1} \mathbf{d}_{\omega_{j}}+s_{2} \mathbf{d}_{\omega_{k}}\right)\right\} .
\end{aligned}
$$

To obtain the joint pdf of $\omega_{j}$ and $\omega_{k}$, the two-dimensional inverse Laplace transform of (24) is required. An exact closed-form expressions for the general case is not possible. Therefore, we calculate the joint cumulants of the natural frequencies.

If first-order perturbation is used then $\mathbf{D}_{\omega_{j}}=\mathbf{D}_{\omega_{k}}=\mathbf{O}$ and from (24) we obtain

$$
M_{\omega_{j}, \omega_{k}}\left(s_{1}, s_{2}\right)=\exp \left\{\boldsymbol{\mu}_{\omega_{j k}}^{T} \widetilde{\mathbf{s}}+\frac{1}{2} \widetilde{\mathbf{s}}^{T} \boldsymbol{\Sigma}_{\omega_{j k}} \widetilde{\mathbf{s}}\right\}
$$


where

$$
\widetilde{\mathbf{s}}=\left\{\begin{array}{c}
s_{1} \\
s_{2}
\end{array}\right\}, \quad \boldsymbol{\mu}_{\omega_{j k}}=\left\{\begin{array}{c}
\bar{\omega}_{j} \\
\bar{\omega}_{k}
\end{array}\right\}, \quad \text { and } \quad \boldsymbol{\Sigma}_{\omega_{j k}}=\left[\begin{array}{lll}
\mathbf{d}_{\omega_{j}}^{T} \boldsymbol{\Sigma} \mathbf{d}_{\omega_{j}} & \mathbf{d}_{\omega_{j}}^{T} \boldsymbol{\Sigma} \mathbf{d}_{\omega_{k}} \\
\mathbf{d}_{\omega_{j}}^{T} \boldsymbol{\Sigma} \mathbf{d}_{\omega_{k}} & \mathbf{d}_{\omega_{k}}^{T} \boldsymbol{\Sigma} \mathbf{d}_{\omega_{k}}
\end{array}\right] .
$$

This implies that $\omega_{j}$ and $\omega_{k}$ are jointly Gaussian-distributed with mean $\boldsymbol{\mu}_{\omega_{j k}}$ and covariance matrix $\boldsymbol{\Sigma}_{\omega_{j k}}$.

For second-order perturbation, the joint cumulants of $\omega_{j}$ and $\omega_{k}$ can be obtained by taking the logarithm of the joint moment generating function (also known as the cumulant generating function),

$$
\begin{array}{r}
\ln M_{\omega_{j}, \omega_{k}}\left(s_{1}, s_{2}\right)=-\frac{1}{2} \ln \left\|\mathbf{I}-s_{1} \mathbf{D}_{\omega_{j}} \boldsymbol{\Sigma}-s_{2} \mathbf{D}_{\omega_{k}} \boldsymbol{\Sigma}\right\|+\left(s_{1} \bar{\omega}_{j}+s_{2} \bar{\omega}_{k}\right) \\
\quad+\frac{1}{2}\left(s_{1} \mathbf{d}_{\omega_{j}}+s_{2} \mathbf{d}_{\omega_{k}}\right)^{T} \boldsymbol{\Sigma}\left[\mathbf{I}-s_{1} \mathbf{D}_{\omega_{j}} \boldsymbol{\Sigma}-s_{2} \mathbf{D}_{\omega_{k}} \boldsymbol{\Sigma}\right]^{-1}\left(s_{1} \mathbf{d}_{\omega_{j}}+s_{2} \mathbf{d}_{\omega_{k}}\right) .
\end{array}
$$

A general $\left(r_{1}, r_{2}\right)$ th order cumulant of the $j$ th and $k$ th natural frequencies can be obtained from

$$
\kappa_{j k}^{\left(r_{1}, r_{2}\right)}=\left.\frac{\partial^{r_{1}+r_{2}}}{\partial s_{1}^{r_{1}} \partial s_{2}^{r_{2}}} \ln M_{\omega_{j}, \omega_{k}}\left(s_{1}, s_{2}\right)\right|_{s_{1}=0, s_{2}=0} .
$$

Suppose $s_{1}$ and $s_{2}$ are small so that all the eigenvalues of the matrix,

$$
\mathbf{C}=s_{1} \mathbf{D}_{\omega_{j}} \boldsymbol{\Sigma}+s_{2} \mathbf{D}_{\omega_{k}} \boldsymbol{\Sigma},
$$

are less than 1 . Then the terms $-\frac{1}{2} \ln \left\|\mathbf{I}-s_{1} \mathbf{D}_{\omega_{j}} \boldsymbol{\Sigma}-s_{2} \mathbf{D}_{\omega_{k}} \boldsymbol{\Sigma}\right\|$ and $\left[\mathbf{I}-s_{1} \mathbf{D}_{\omega_{j}} \boldsymbol{\Sigma}-s_{2} \mathbf{D}_{\omega_{k}} \boldsymbol{\Sigma}\right]^{-1}$ appearing in (27) can be expanded in a Taylor series as

$$
\begin{aligned}
-\frac{1}{2} \ln \|\mathbf{I}-\mathbf{C}\| & =\operatorname{Trace}(\mathbf{C})+\frac{1}{2} \operatorname{Trace}\left(\mathbf{C}^{2}\right)+\frac{1}{3} \operatorname{Trace}\left(\mathbf{C}^{3}\right)+\cdots \\
\text { and } \quad[\mathbf{I}-\mathbf{C}]^{-1} & =\mathbf{I}+\mathbf{C}+\mathbf{C}^{2}+\cdots .
\end{aligned}
$$

Using these expansions, the cumulant generating function in (27) can be expressed by a polynomial in $s_{1}$ and $s_{2}$ and therefore the cumulants can be obtained from (28) in a straightforward manner. After some simplifications the following results can be derived

$$
\begin{aligned}
\kappa_{j k}^{(1,0)} & =\mathrm{E}\left[\omega_{j}\right]=\bar{\omega}_{j}+\frac{1}{2} \operatorname{Trace}\left(\mathbf{D}_{\omega_{j}} \boldsymbol{\Sigma}\right), \\
\kappa_{j k}^{(0,1)} & =\mathrm{E}\left[\omega_{k}\right]=\bar{\omega}_{k}+\frac{1}{2} \operatorname{Trace}\left(\mathbf{D}_{\omega_{k}} \boldsymbol{\Sigma}\right), \\
\kappa_{j k}^{(1,1)} & =\operatorname{Cov}\left(\omega_{j}, \omega_{k}\right)=\frac{1}{2} \operatorname{Trace}\left(\left(\mathbf{D}_{\omega_{j}} \boldsymbol{\Sigma}\right)\left(\mathbf{D}_{\omega_{k}} \boldsymbol{\Sigma}\right)\right)+\mathbf{d}_{\omega_{j}}^{T} \boldsymbol{\Sigma} \mathbf{d}_{\omega_{k}} .
\end{aligned}
$$

The general case can be obtained following Mathai \& Provost (1992, chap. 3) as

$$
\begin{aligned}
\kappa_{j k}^{\left(r_{1}, r_{2}\right)}= & \frac{1}{2}\left(r_{1}+r_{2}-2\right) !\left\{\left(r_{1}+r_{2}-1\right) \operatorname{Trace}\left(\left(\mathbf{D}_{\omega_{j}} \boldsymbol{\Sigma}\right)^{r_{1}}\left(\mathbf{D}_{\omega_{k}} \boldsymbol{\Sigma}\right)^{r_{2}}\right)\right. \\
& +r_{1}\left(r_{1}-1\right) \mathbf{d}_{\omega_{j}}^{T}\left(\mathbf{D}_{\omega_{j}} \boldsymbol{\Sigma}\right)^{r_{1}-2}\left(\mathbf{D}_{\omega_{k}} \boldsymbol{\Sigma}\right)^{r_{2}} \boldsymbol{\Sigma} \mathbf{d}_{\omega_{j}} \\
& +r_{2}\left(r_{2}-1\right) \mathbf{d}_{\omega_{k}}^{T}\left(\mathbf{D}_{\omega_{j}} \boldsymbol{\Sigma}\right)^{r_{1}}\left(\mathbf{D}_{\omega_{k}} \boldsymbol{\Sigma}\right)^{r_{2}-2} \boldsymbol{\Sigma} \mathbf{d}_{\omega_{k}} \\
& \left.+2 r_{1} r_{2} \mathbf{d}_{\omega_{j}}^{T}\left(\mathbf{D}_{\omega_{j}} \boldsymbol{\Sigma}\right)^{r_{1}-1}\left(\mathbf{D}_{\omega_{k}} \boldsymbol{\Sigma}\right)^{r_{2}-1} \boldsymbol{\Sigma} \mathbf{d}_{\omega_{k}}\right\}, \quad \text { for } \quad r_{1} \geq 1, r_{2} \geq 1 .
\end{aligned}
$$


Since all the cumulants are known from the preceding expressions, the joint pdf of the natural frequencies can be calculated, for example using Edgeworth expansion, up to any desired accuracy. Recall that the limitation of this approach arises from the second-order Taylor expansion of the natural frequencies around the deterministic values. Therefore, the inclusion of higher order cumulants in the expression of the joint pdf will not overcome this fundamental limitation.

The method described in this section is only applicable when the basic random variables are Gaussian. When the elements of $\mathbf{x}$ are non-Gaussian then neither the first-order perturbation nor the second-order perturbation methods are helpful because there is no general method to obtain the resulting statistics in a simple manner. In such cases the method outlined in the next section might be useful.

\section{Statistics of the natural frequencies using asymptotic integral based method}

\subsection{Multi-dimensional integrals in unbounded domains}

In this section the moments of the natural frequencies are obtained based on an asymptotic approximation of the multi-dimensional integral. Consider a function $f(\mathbf{x}): \mathbb{R}^{m} \mapsto \mathbb{R}$ which is smooth and at least twice differentiable. Suppose we want to evaluate an integral of the following form:

$$
\mathcal{J}=\int_{\mathbb{R}^{m}} \exp \{-f(\mathbf{x})\} \mathrm{d} \mathbf{x} .
$$

This is an $m$-dimensional integral over the unbounded domain $\mathbb{R}^{m}$. The maximum contribution to this integral comes from the neighborhood where $f(\mathbf{x})$ reaches its global minimum. Suppose that $f(\mathbf{x})$ reaches its global minimum at an unique point $\boldsymbol{\theta} \in \mathbb{R}^{m}$. Therefore, at $\mathbf{x}=\boldsymbol{\theta}$

$$
\partial f(\mathbf{x}) / \partial x_{k}=0, \forall k \quad \text { or } \quad \mathbf{d}_{f}(\boldsymbol{\theta})=\mathbf{0} .
$$

Using this, expand $f(\mathbf{x})$ in a Taylor series about $\boldsymbol{\theta}$ and rewrite (36) as

$$
\begin{aligned}
\mathcal{J} & =\int_{\mathbb{R}^{m}} \exp \left\{-\left\{f(\boldsymbol{\theta})+\frac{1}{2}(\mathbf{x}-\boldsymbol{\theta})^{T} \mathbf{D}_{f}(\boldsymbol{\theta})(\mathbf{x}-\boldsymbol{\theta})+\varepsilon(\mathbf{x}, \boldsymbol{\theta})\right\}\right\} \mathrm{d} \mathbf{x} \\
& =\exp \{-f(\boldsymbol{\theta})\} \int_{\mathbb{R}^{m}} \exp \left\{-\frac{1}{2}(\mathbf{x}-\boldsymbol{\theta})^{T} \mathbf{D}_{f}(\boldsymbol{\theta})(\mathbf{x}-\boldsymbol{\theta})-\varepsilon(\mathbf{x}, \boldsymbol{\theta})\right\} \mathrm{d} \mathbf{x},
\end{aligned}
$$

where $\varepsilon(\mathbf{x}, \boldsymbol{\theta})$ is the error only if the terms up to second-order were retained in the Taylor series expansion. With suitable scaling of $\mathbf{x}$ the integral in (36) can be transformed to the socalled 'Laplace integral'. Under special conditions such integrals can be well approximated using asymptotic methods. The relevant mathematical methods and formal derivations are covered in detail in the books by Bleistein \& Handelsman (1994) and Wong (2001). Here we propose a slightly different version of asymptotic integrals. The error $\varepsilon(\mathbf{x}, \boldsymbol{\theta})$ depends on the higher order derivatives of $f(\mathbf{x})$ at $\mathbf{x}=\boldsymbol{\theta}$. If they are small compared to $f(\boldsymbol{\theta})$ and the elements of $\mathbf{D}_{f}(\boldsymbol{\theta})$, their contribution to the value of the integral will be negligible. Therefore, we assume that $f(\boldsymbol{\theta})$ and the elements of $\mathbf{D}_{f}(\boldsymbol{\theta})$ are large, so that

$$
\left|\frac{1}{f(\boldsymbol{\theta})} \mathrm{D}^{(j)}(f(\boldsymbol{\theta}))\right| \rightarrow 0 \text { and } \forall k, l,\left|\frac{1}{\left[\mathbf{D}_{f}(\boldsymbol{\theta})\right]_{k l}} \mathrm{D}^{(j)}(f(\boldsymbol{\theta}))\right| \rightarrow 0, \text { for } j>2 \text {, }
$$


where $\mathrm{D}^{(j)}(f(\boldsymbol{\theta}))$ is the $j$ th order derivative of $f(\mathbf{x})$ evaluated at $\mathbf{x}=\boldsymbol{\theta}$. Under such assumptions $\varepsilon(\mathbf{x}, \boldsymbol{\theta}) \rightarrow 0$. Therefore, the integral in (38) can be approximated as

$$
\mathcal{J} \approx \exp \{-f(\boldsymbol{\theta})\} \int_{\mathbb{R}^{m}} \exp \left\{-\frac{1}{2}(\mathbf{x}-\boldsymbol{\theta})^{T} \mathbf{D}_{f}(\boldsymbol{\theta})(\mathbf{x}-\boldsymbol{\theta})\right\} \mathrm{d} \mathbf{x} .
$$

If $\boldsymbol{\theta}$ is the global minima of $f(\mathbf{x})$ in $\mathbb{R}^{m}$, the symmetric Hessian matrix $\mathbf{D}_{f}(\boldsymbol{\theta}) \in \mathbb{R}^{m \times m}$ is also expected to be positive definite. Now use the coordinate transformation

$$
\boldsymbol{\xi}=(\mathbf{x}-\boldsymbol{\theta}) \mathbf{D}_{f}^{-1 / 2}(\boldsymbol{\theta}) .
$$

The Jacobian of this transformation is

$$
\|\mathbf{J}\|=\left\|\mathbf{D}_{f}(\boldsymbol{\theta})\right\|^{-1 / 2} .
$$

Using (41), the integral in (40) can be evaluated as

$$
\begin{aligned}
\mathcal{J} & \approx \exp \{-f(\boldsymbol{\theta})\} \int_{\mathbb{R}^{m}}\left\|\mathbf{D}_{f}(\boldsymbol{\theta})\right\|^{-1 / 2} \exp \left\{-\frac{1}{2}\left(\boldsymbol{\xi}^{T} \boldsymbol{\xi}\right)\right\} \mathrm{d} \boldsymbol{\xi}, \\
\text { or } \quad \mathcal{J} & \approx(2 \pi)^{m / 2} \exp \{-f(\boldsymbol{\theta})\}\left\|\mathbf{D}_{f}(\boldsymbol{\theta})\right\|^{-1 / 2} .
\end{aligned}
$$

The analysis proposed here is slightly different from the classical Laplace method of asymptotic approximation of integrals (Wong 2001, chap. 9, theorem 3). Here it is simply assumed that the higher order derivatives $\mathrm{D}^{(j)}(f(\boldsymbol{\theta}))$ are negligibly small compared to $f(\boldsymbol{\theta})$ and the elements of $\mathbf{D}_{f}(\boldsymbol{\theta})$. This approximation is expected to yield good results if the minima of $f(\mathbf{x})$ around $\mathbf{x}=\boldsymbol{\theta}$ is sharp. If $\boldsymbol{\theta}$ is not unique then it is required to sum the contributions arising from all such optimal points separately. Equation (44) will now be used to obtain joint moments of the natural frequencies.

\subsection{Joint moments of two natural frequencies}

A general $\left(r_{1}, r_{2}\right)$ th order joint moment of two natural frequencies $\omega_{j}$ and $\omega_{k}$ can be expressed as

$$
\begin{aligned}
\mu_{j k}^{\left(r_{1}, r_{2}\right)}= & \mathrm{E}\left[\omega_{j}^{r_{1}}(\mathbf{x}) \omega_{k}^{r_{2}}(\mathbf{x})\right]=\int_{\mathbb{R}^{m}} \omega_{j}^{r_{1}}(\mathbf{x}) \omega_{k}^{r_{2}}(\mathbf{x}) p_{\mathbf{x}}(\mathbf{x}) \mathrm{d} \mathbf{x} \\
= & \int_{\mathbb{R}^{m}} \exp \left\{-\left(L(\mathbf{x})-r_{1} \ln \omega_{j}(\mathbf{x})-r_{2} \ln \omega_{k}(\mathbf{x})\right)\right\} \mathrm{d} \mathbf{x}, \\
& r_{1}, r_{2}=1,2,3 \ldots
\end{aligned}
$$

The equation can be expressed in the form of (36) by choosing

$$
f(\mathbf{x})=L(\mathbf{x})-r_{1} \ln \omega_{j}(\mathbf{x})-r_{2} \ln \omega_{k}(\mathbf{x}) .
$$

Differentiating the above equation with respect to $x_{i}$ we obtain

$$
\frac{\partial f(\mathbf{x})}{\partial x_{i}}=\frac{\partial L(\mathbf{x})}{\partial x_{i}}-\frac{r_{1}}{\omega_{j}(\mathbf{x})} \frac{\partial \omega_{j}(\mathbf{x})}{\partial x_{i}}-\frac{r_{2}}{\omega_{k}(\mathbf{x})} \frac{\partial \omega_{k}(\mathbf{x})}{\partial x_{i}} .
$$


The optimal point $\boldsymbol{\theta}$ can be obtained from (37) by equating the above expression to zero. Therefore at $\mathbf{x}=\boldsymbol{\theta}$

$$
\begin{aligned}
& \frac{\partial f(\mathbf{x})}{\partial x_{i}}=0, \quad \forall i, \\
& \text { or } \quad \frac{r_{1}}{\omega_{j}(\mathbf{x})} \frac{\partial \omega_{j}(\mathbf{x})}{\partial x_{i}}+\frac{r_{2}}{\omega_{k}(\mathbf{x})} \frac{\partial \omega_{k}(\mathbf{x})}{\partial x_{i}}=\frac{\partial L(\boldsymbol{\theta})}{\partial x_{i}}, \quad \forall k, \\
& \text { or } \quad \mathbf{d}_{L}(\boldsymbol{\theta})=\frac{r_{1}}{\omega_{j}(\boldsymbol{\theta})} \mathbf{d}_{\omega_{j}}(\boldsymbol{\theta})+\frac{r_{2}}{\omega_{k}(\boldsymbol{\theta})} \mathbf{d}_{\omega_{k}}(\boldsymbol{\theta}) .
\end{aligned}
$$

The elements of the Hessian matrix $\mathbf{D}_{f}(\boldsymbol{\theta})$ can be obtained by differentiating (47) with respect to $x_{l}$ :

$$
\begin{aligned}
\frac{\partial^{2} f(\mathbf{x})}{\partial x_{i} \partial x_{l}}= & \frac{\partial^{2} L(\mathbf{x})}{\partial x_{i} \partial x_{l}}+\frac{r_{1}}{\omega_{j}^{2}(\mathbf{x})} \frac{\partial \omega_{j}(\mathbf{x})}{\partial x_{l}} \frac{\partial \omega_{j}(\mathbf{x})}{\partial x_{i}}-\frac{r_{1}}{\omega_{j}(\mathbf{x})} \frac{\partial^{2} \omega_{j}(\mathbf{x})}{\partial x_{i} \partial x_{l}} \\
& +\frac{r_{2}}{\omega_{k}^{2}(\mathbf{x})} \frac{\partial \omega_{k}(\mathbf{x})}{\partial x_{l}} \frac{\partial \omega_{k}(\mathbf{x})}{\partial x_{i}}-\frac{r_{2}}{\omega_{k}(\mathbf{x})} \frac{\partial^{2} \omega_{k}(\mathbf{x})}{\partial x_{i} \partial x_{l}}
\end{aligned}
$$

Combining this equation for all $i$ and $l$ we have

$$
\begin{aligned}
\mathbf{D}_{f}(\boldsymbol{\theta})= & \mathbf{D}_{L}(\boldsymbol{\theta})+\frac{r_{1}}{\omega_{j}^{2}(\boldsymbol{\theta})} \mathbf{d}_{\omega_{j}}(\boldsymbol{\theta}) \mathbf{d}_{\omega_{j}}^{T}(\boldsymbol{\theta})-\frac{r_{1}}{\omega_{j}(\boldsymbol{\theta})} \mathbf{D}_{\omega_{j}}(\boldsymbol{\theta}) \\
& +\frac{r_{2}}{\omega_{k}^{2}(\boldsymbol{\theta})} \mathbf{d}_{\omega_{k}}(\boldsymbol{\theta}) \mathbf{d}_{\omega_{k}}^{T}(\boldsymbol{\theta})-\frac{r_{2}}{\omega_{k}(\boldsymbol{\theta})} \mathbf{D}_{\omega_{k}}(\boldsymbol{\theta}) .
\end{aligned}
$$

Using the asymptotic approximation (44), the joint moment of two natural frequencies can be obtained as

$$
\mu_{j k}^{\left(r_{1}, r_{2}\right)} \approx(2 \pi)^{m / 2} \omega_{j}^{r_{1}}(\boldsymbol{\theta}) \omega_{k}^{r_{2}}(\boldsymbol{\theta}) \exp \{-L(\boldsymbol{\theta})\}\left\|\mathbf{D}_{f}(\boldsymbol{\theta})\right\|^{-1 / 2} .
$$

The mean of the natural frequencies can be obtained by substituting $r_{1}=0$ or $r_{2}=0$ in (53) as

$$
\mathrm{E}\left[\omega_{j}\right]=\mu_{j k}^{(1,0)} \approx(2 \pi)^{m / 2} \omega_{j}(\boldsymbol{\theta}) \exp \{-L(\boldsymbol{\theta})\}\left\|\mathbf{D}_{f}(\boldsymbol{\theta})\right\|^{-1 / 2},
$$

where $\theta$ is obtained from

$$
\begin{aligned}
\mathbf{d}_{L}(\boldsymbol{\theta}) & =\mathbf{d}_{\omega_{j}}(\boldsymbol{\theta}) / \omega_{j}(\boldsymbol{\theta}) \\
\text { and } \quad \mathbf{D}_{f}(\boldsymbol{\theta}) & =\mathbf{D}_{L}(\boldsymbol{\theta})+\left(\mathbf{d}_{\omega_{j}}(\boldsymbol{\theta}) \mathbf{d}_{\omega_{j}}^{T}(\boldsymbol{\theta}) / \omega_{j}(\boldsymbol{\theta})-\mathbf{D}_{\omega_{j}}(\boldsymbol{\theta})\right) / \omega_{j}(\boldsymbol{\theta}) .
\end{aligned}
$$

The elements of the covariance matrix of the natural frequencies can obtained as

$$
\operatorname{Cov}\left(\omega_{j}, \omega_{k}\right)=\mathrm{E}\left[\left(\omega_{j}-\mathrm{E}\left[\omega_{j}\right]\right)\left(\omega_{k}-\mathrm{E}\left[\omega_{k}\right]\right)\right]=\mu_{j k}^{(1,1)}-\mu_{j k}^{(1,0)} \mu_{j k}^{(0,1)} .
$$

The optimal point $\boldsymbol{\theta}$ should be calculated by solving the nonlinear set of equations (50). Since the explicit analytical expression of $\mathbf{d}_{\omega_{j}}$ in terms of the derivative of the mass and stiffness matrices is available (see appendix A), expensive numerical differentiation of $\omega_{j}(\mathbf{x})$ at each step is not needed. Moreover, for most $p_{\mathbf{x}}(\mathbf{x})$, a closed-form expression of $\mathbf{d}_{L}(\mathbf{x})$ is available. 
We illustrate a special case when $\mathbf{x}$ is distributed as multivariate Gaussian vector. In this case $L(\mathbf{x})$ is given by (3), and by differentiating (3) successively we obtain

$$
\begin{array}{rlrl}
\mathbf{d}_{L}(\mathbf{x}) & =\boldsymbol{\Sigma}^{-1}(\mathbf{x}-\boldsymbol{\mu}) \\
\text { and } & \mathbf{D}_{L}(\mathbf{x}) & =\boldsymbol{\Sigma}^{-1} .
\end{array}
$$

The optimal point $\boldsymbol{\theta}$ can be obtained from (50) as

$$
\boldsymbol{\theta}=\boldsymbol{\mu}+\boldsymbol{\Sigma}\left[\frac{r_{1}}{\omega_{j}(\boldsymbol{\theta})} \mathbf{d}_{\omega_{j}}(\boldsymbol{\theta})+\frac{r_{2}}{\omega_{k}(\boldsymbol{\theta})} \mathbf{d}_{\omega_{k}}(\boldsymbol{\theta})\right] .
$$

This equation can be used to obtain $\boldsymbol{\theta}$ in an iterative manner. We start with an initial guess of $\boldsymbol{\theta}=\boldsymbol{\mu}$ on the right-hand side and obtain an updated $\boldsymbol{\theta}$ on the left-hand side. This procedure can be continued till the difference between the values of $\boldsymbol{\theta}$ obtained from both sides of (60) is less than a predefined small value (any vector norm can be used to measure the difference).

\subsection{Arbitrary order joint moments of multiple natural frequencies}

The formulation presented in the previous subsection can be readily generalized to obtain arbitrary order joint moments of multiple natural frequencies. We want to obtain

$$
\mu_{j_{1} j_{2} \ldots j_{n}}^{\left(r_{1}, r_{2}, \ldots r_{n}\right)}=\int_{\mathbb{R}^{m}}\left\{\omega_{j_{1}}^{r_{1}}(\mathbf{x}) \omega_{j_{2}}^{r_{2}}(\mathbf{x}) \ldots \omega_{j_{n}}^{r_{n}}(\mathbf{x})\right\} p_{\mathbf{x}}(\mathbf{x}) \mathrm{d} \mathbf{x} .
$$

By choosing the function

$$
f(\mathbf{x})=L(\mathbf{x})-r_{1} \ln \omega_{j_{1}}(\mathbf{x})-r_{2} \ln \omega_{j_{2}}(\mathbf{x})-\cdots r_{n} \ln \omega_{j_{n}}(\mathbf{x})
$$

and applying the asymptotic approximation in (44), it can be shown that

$$
\mu_{j_{1} j_{2} \ldots j_{n}}^{\left(r_{1}, r_{2}, \ldots r_{n}\right)} \approx(2 \pi)^{m / 2}\left\{\omega_{j_{1}}^{r_{1}}(\boldsymbol{\theta}) \omega_{j_{2}}^{r_{2}}(\boldsymbol{\theta}) \ldots \omega_{j_{n}}^{r_{n}}(\boldsymbol{\theta})\right\} \exp \{-L(\boldsymbol{\theta})\}\left\|\mathbf{D}_{f}(\boldsymbol{\theta})\right\|^{-1 / 2},
$$

where $\theta$ is obtained by solving

$$
\mathbf{d}_{L}(\boldsymbol{\theta})=\frac{r_{1}}{\omega_{j_{1}}(\boldsymbol{\theta})} \mathbf{d}_{\omega_{j_{1}}}(\boldsymbol{\theta})+\frac{r_{2}}{\omega_{j_{2}}(\boldsymbol{\theta})} \mathbf{d}_{\omega_{j_{2}}}(\boldsymbol{\theta})+\cdots \frac{r_{n}}{\omega_{j_{n}}(\boldsymbol{\theta})} \mathbf{d}_{\omega_{j_{n}}}(\boldsymbol{\theta}),
$$

and the Hessian matrix is given by

$$
\mathbf{D}_{f}(\boldsymbol{\theta})=\mathbf{D}_{L}(\boldsymbol{\theta})+\sum_{\substack{j=j_{1}, j_{2}, \ldots \\ r=r_{1}, r_{2}, \ldots}}^{j_{n}, r_{n}} \frac{r}{\omega_{j}^{2}(\boldsymbol{\theta})} \mathbf{d}_{\omega_{j}}(\boldsymbol{\theta}) \mathbf{d}_{\omega_{j}}^{T}(\boldsymbol{\theta})-\frac{r}{\omega_{j}(\boldsymbol{\theta})} \mathbf{D}_{\omega_{j}}(\boldsymbol{\theta}) .
$$

Equation (63) is perhaps the most general formula to obtain the moments of the natural frequencies of linear stochastic dynamic systems. Once the joint moments are known, the joint probability density functions of the natural frequencies are obtained, for example, using the maximum entropy principle. The proposed method is applied to dynamic analysis of a 3-dof system with uncertain properties. 


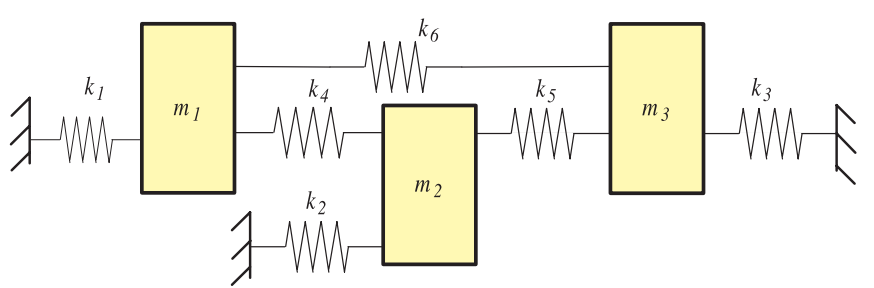

Figure 1. The three-degree-offreedom random system.

\section{Numerical examples}

\subsection{System model and computational methodology}

A three-degree-of-freedom (3-dof) undamped spring-mass system, taken from Friswell (1996), is shown in figure 1. The mass and stiffness matrices of the example system are given by

$$
\mathbf{M}=\left[\begin{array}{ccc}
m_{1} & 0 & 0 \\
0 & m_{2} & 0 \\
0 & 0 & m_{3}
\end{array}\right] \text { and } \mathbf{K}=\left[\begin{array}{ccc}
k_{1}+k_{4}+k_{6} & -k_{4} & -k_{6} \\
-k_{4} & k_{4}+k_{5}+k_{6} & -k_{5} \\
-k_{6} & -k_{5} & k_{5}+k_{3}+k_{6}
\end{array}\right]
$$

It is assumed that all mass and stiffness constants are random. The randomness in these parameters are assumed to be of the following form:

$$
\begin{aligned}
m_{i} & =\bar{m}_{i}\left(1+\epsilon_{m} x_{i}\right), \quad i=1,2,3, \\
k_{i} & =\bar{k}_{i}\left(1+\epsilon_{k} x_{i+3}\right), \quad i=1, \ldots, 6 .
\end{aligned}
$$

Here $\mathbf{x}=\left\{x_{1}, \ldots, x_{9}\right\}^{T} \in \mathbb{R}^{9}$ is the vector of random variables. It is assumed that all random variables are Gaussian and uncorrelated with zero mean and unit standard deviation, that is $\boldsymbol{\mu}=\mathbf{0}$ and $\boldsymbol{\Sigma}=\mathbf{I}$. Therefore, the mean values of $m_{i}$ and $k_{i}$ are given by $\bar{m}_{i}$ and $\bar{k}_{i}$. The numerical values of the constants are assumed to be $\bar{m}_{i}=1.0 \mathrm{~kg}$ for $i=1,2,3$; $\bar{k}_{i}=1.0 \mathrm{~N} / \mathrm{m}$ for $i=1, \ldots, 5$ and $k_{6}=3.0 \mathrm{~N} / \mathrm{m}$. The numerical values of the 'strength parameters' are assumed to be $\epsilon_{m}=0.15$ and $\epsilon_{k}=0.20$. In order to obtain the statistics of the natural frequencies using the methods developed in this paper, the gradient vector and the Hessian matrix of the natural frequencies are required. As shown in appendix A, this in turn requires the derivative of the system matrices with respect to the entries of $\mathbf{x}$. For most practical problems, which usually involve finite element modelling, these derivatives have to be determined numerically. However, for this simple example the derivatives can be obtained in closed-form and they are given in appendix B.

We calculate joint moments and joint probability density functions of the natural frequencies of the system. Attention is restricted up to second-order joint statistics of two natural frequencies. The following four methods are used to obtain the joint moments and the joint probability density functions:

(1) First-order perturbation: For this case the mean and covariance matrix of the natural frequencies are calculated using (32) and (34) by substituting the Hessian matrices $\mathbf{D}_{\omega_{j}}=\mathbf{O}$ 
and $\mathbf{D}_{\omega_{k}}=\mathbf{O}$. Recalling that for this problem $\boldsymbol{\Sigma}=\mathbf{I}$, the resulting statistics for this special case can be obtained as

$$
\begin{aligned}
\mathrm{E}[\omega]_{j} & =\bar{\omega}_{j} \\
\text { and } \quad \operatorname{Cov}\left(\omega_{j}, \omega_{k}\right) & =\mathbf{d}_{\omega_{j}}^{T} \mathbf{d}_{\omega_{k}} .
\end{aligned}
$$

The gradient vector $\mathbf{d}_{\omega_{j}}$ can be obtained from (A2) using the system derivative matrices (B1) and (B2).

(2) Second-order perturbation: In this case, the Hessian matrices $\mathbf{D}_{\omega_{i}}$ and $\mathbf{D}_{\omega_{k}}$ are used in calculating the joint statistics of the natural frequencies using (32) and (34). The elements of the Hessian matrices $\mathbf{D}_{\omega_{j}}$ and $\mathbf{D}_{\omega_{k}}$ can be calculated using (A4). The resulting statistics for this special case can be obtained as

$$
\begin{aligned}
\mathrm{E}[\omega]_{j} & =\bar{\omega}_{j}+\frac{1}{2} \operatorname{Trace}\left(\mathbf{D}_{\omega_{j}}\right) \\
\text { and } \operatorname{Cov}\left(\omega_{j}, \omega_{k}\right) & =\mathbf{d}_{\omega_{j}}^{T} \mathbf{d}_{\omega_{k}}+\frac{1}{2} \operatorname{Trace}\left(\mathbf{D}_{\omega_{j}} \mathbf{D}_{\omega_{k}}\right)
\end{aligned}
$$

Comparing these results with (69) and (70), the contributions of the Hessian matrices can be regarded as corrections to the first-order perturbation results.

(3) Method based on the asymptotic integral: In this case the mean and covariance matrix of the natural frequencies are calculated using (54) and (57). The function $L(\mathbf{x})$ can be obtained by substituting $\boldsymbol{\mu}=\mathbf{0}$ and $\boldsymbol{\Sigma}=\mathbf{I}$ in (3) as

$$
L(\mathbf{x})=\frac{m}{2} \ln (2 \pi)+\frac{1}{2} \mathbf{x}^{T} \mathbf{x} .
$$

The gradient vector and the Hessian matrix of $L(\mathbf{x})$, needed to apply this method, are given by

$$
\mathbf{d}_{L}(\mathbf{x})=\mathbf{x} \quad \text { and } \quad \mathbf{D}_{L}(\mathbf{x})=\mathbf{I} .
$$

(4) Monte Carlo simulation (MCS): The samples of the nine independent Gaussian random variables $x_{i}, i=1, \ldots, 9$ are generated and the natural frequencies are computed directly from (1). A total of 15000 samples are used to obtain the statistical moments and histograms of the pdf of the natural frequencies. The results obtained from MCS are assumed to be the benchmark for the purpose of comparing the analytical methods.

The results are presented and discussed in the next subsection.

\subsection{Numerical results}

For the given parameter values the natural frequencies (in $\mathrm{rad} / \mathrm{s}$ ) of the corresponding deterministic system is given by

$$
\bar{\omega}_{1}=1, \quad \bar{\omega}_{2}=2, \quad \text { and } \quad \bar{\omega}_{3}=3 .
$$

Figure 2 shows percentage error with respect to Monte Carlo simulation in the elements of the mean vector and covariance matrix of the natural frequencies.

Since the covariance matrix is a symmetric matrix, only the elements of the upper triangular part are considered for plotting. For the mean values, the first-order perturbation method is 

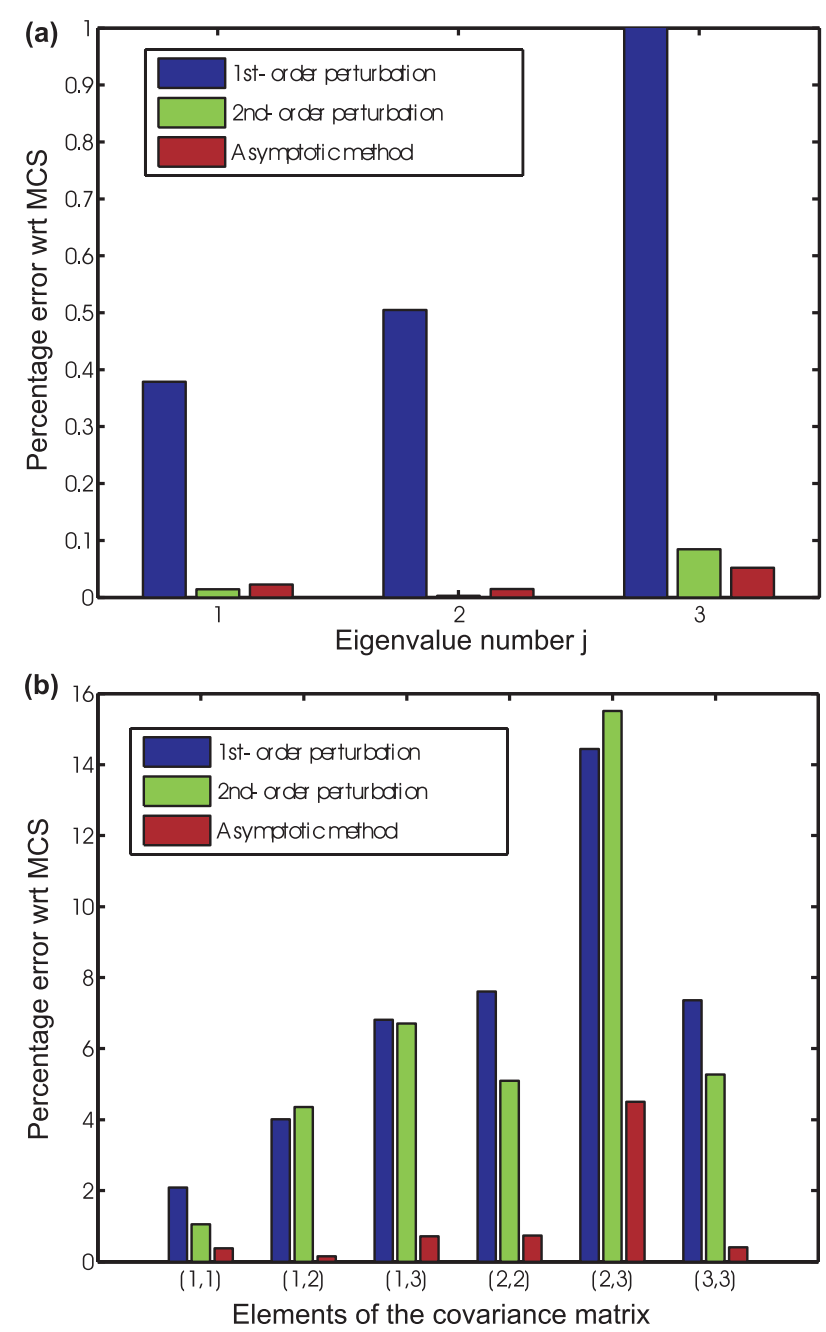

Figure 2. Percentage error with respect to MCS in the mean and covariance matrix of the natural frequencies.

the least accurate, followed by the second-order perturbation method. The same fact is also true for the diagonal elements of the covariance matrix (that is the variance of the natural frequencies). However, for the off-diagonal terms, the second-order perturbation method appears to be slightly less accurate compared to the first-order perturbation method. For both calculations, the asymptotic method is clearly the most accurate among the three analytical methods used in this study.

Now consider the probability density function of the natural frequencies. Because the asymptotic method is the most accurate among the three methods discussed here, we will only pursue this method in the remaining discussions. First, we focus on the marginal pdf of the natural frequencies. Using the asymptotic method, the mean and standard deviation of the natural frequencies are obtained as

$$
\text { and } \begin{aligned}
\mu_{\Omega} & =\{0.9962,2.0102,3.0312\}^{T} \\
\sigma_{\Omega} & =\{0.0729,0.1603,0.2955\}^{T} .
\end{aligned}
$$




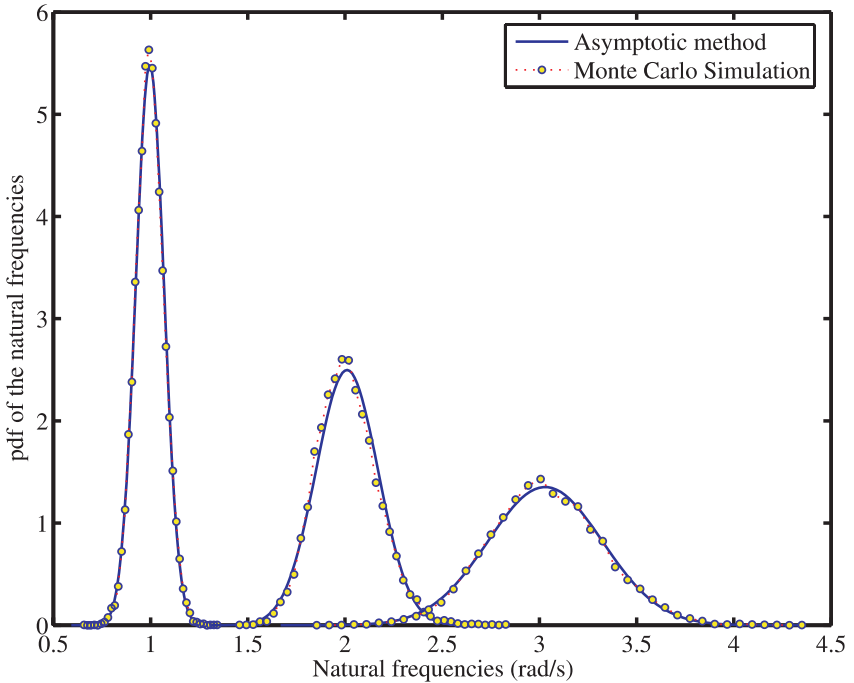

Figure 3. Probability density function of the natural frequencies.

Gaussian distributions are fitted with these parameters and compared with Monte Carlo simulation. The marginal pdf of the natural frequencies obtained from the asymptotic method and Monte Carlo simulation are shown in figure 3.

Each MCS pdf in figure 3 is obtained by normalizing the histogram of the samples so that the area under the curve obtained by joining the middle points of the histogram bins is equal to unity. The Gaussian distributions calculated from the asymptotic method fit the Monte Carlo simulation quite well. This result implies that the probability density function of the individual natural frequencies can be approximated well using a Gaussian distribution with the correct set of parameters.

Now we focus on the joint distribution of the natural frequencies. The covariance matrix and the matrix of correlation coefficients are obtained using the asymptotic method as

$$
\boldsymbol{\Sigma}_{\Omega}=\left[\begin{array}{lll}
0.5319 & 0.5643 & 0.7228 \\
0.5643 & 2.5705 & 0.9821 \\
0.7228 & 0.9821 & 8.7292
\end{array}\right] \times 10^{-2}
$$

and

$$
\rho_{\Omega}=\left[\begin{array}{lll}
1.0000 & 0.4826 & 0.3355 \\
0.4826 & 1.0000 & 0.2073 \\
0.3355 & 0.2073 & 1.0000
\end{array}\right] .
$$

This indicates that the natural frequencies are moderately correlated. The correlation between $\omega_{1}$ and $\omega_{2}$ is more than that between $\omega_{1}$ and $\omega_{3}$. This is expected because $\omega_{3}$ is more distant from $\omega_{1}$, than $\omega_{2}$. However, the correlation between $\omega_{1}$ and $\omega_{3}$ is more than that between $\omega_{2}$ and $\omega_{3}$ in spite of $\omega_{1}$ being further from $\omega_{3}$ as compared to $\omega_{2}$.

In line with the univariate Gaussian distributions shown in figure 3, we can obtain bivariate Gaussian distribution for each pair of natural frequencies.

Joint probability density function of the natural frequencies obtained from the asymptotic method and Monte Carlo simulation are shown in figures 4 and 5. In all, three joint distributions, namely $p_{\omega_{1}, \omega_{2}}, p_{\omega_{1}, \omega_{3}}$ and $p_{\omega_{2}, \omega_{3}}$, are shown in figures 4 and 5 . Each analytical joint pdf 


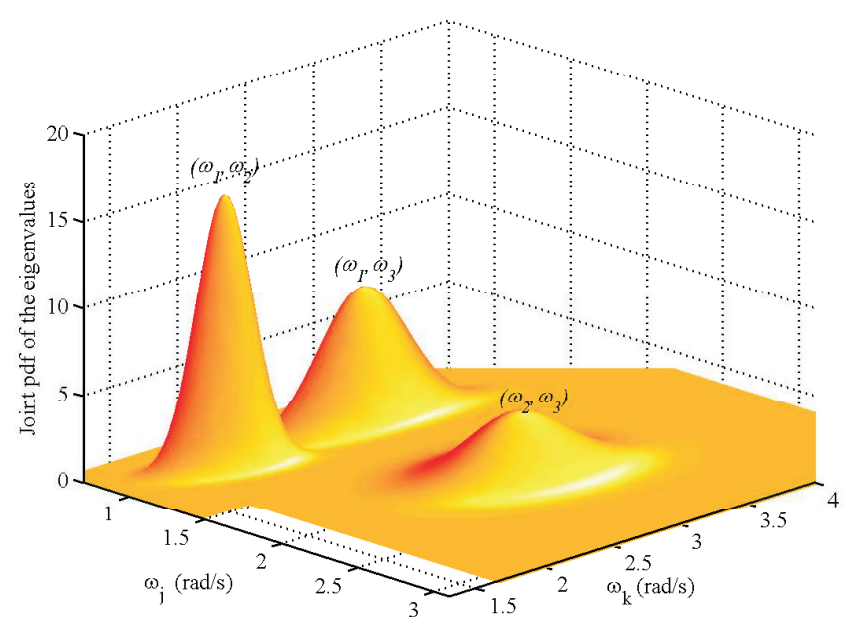

Figure 4. Fitted joint Gaussian probability density function of the natural frequencies using asymptotic method.

in figure 4 is obtained by fitting a bivariate Gaussian distribution with the mean vector and covariance matrix taken from (76) and (78) for the corresponding set of natural frequencies. The MCS pdf in figure 5 is obtained by normalizing the two-dimensional histogram of the samples so that the volume under the surface obtained by joining the middle points of the histogram bins is equal to unity. At first it may appear that, like the marginal pdfs in figure 3 , the joint pdfs of the natural frequencies are also jointly Gaussian-distributed. However, a closer inspection reveals that this is not always the case.

Figure 6 compares the contours of the analytical joint pdf with that obtained from MCS. The adjacent natural frequencies, that is, $\omega_{1}$ and $\omega_{2}$, and $\omega_{2}$ and $\omega_{3}$, are not jointly Gaussiandistributed as the contours of the analytical joint pdf are quite different from that obtained using MCS. The joint pdf of $\omega_{1}$ and $\omega_{3}$ is however close to a bivariate Gaussian density function. The

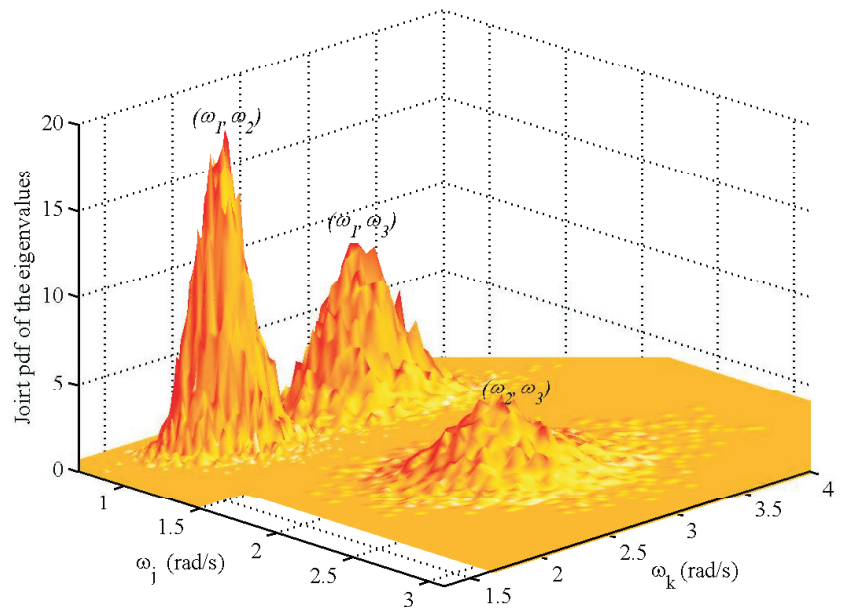

Figure 5. Joint probability density function of the natural frequencies from Monte Carlo simulation. 


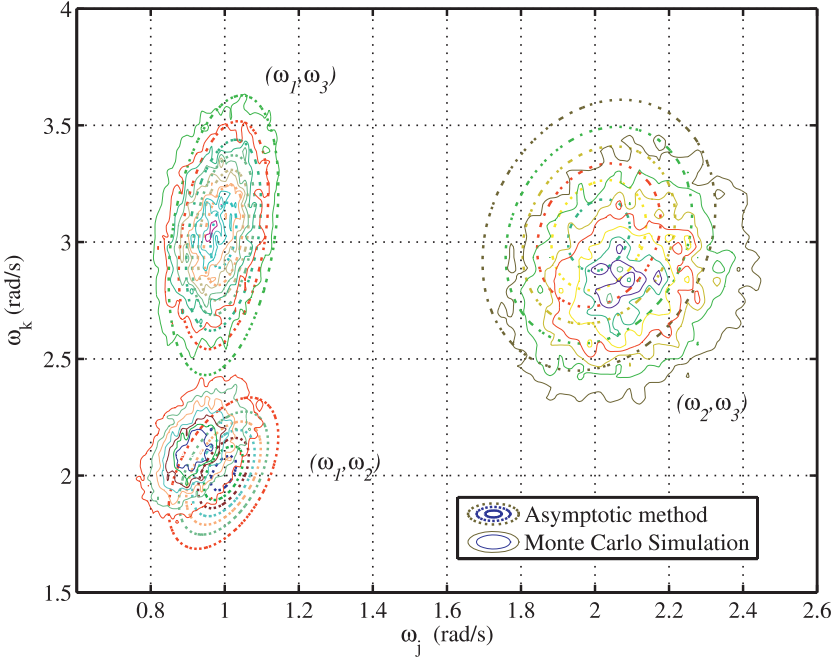

Figure 6. Contours of the joint probability density function of the natural frequencies.

important conclusion that can be drawn from these limited numerical results is that the natural frequencies are in general not jointly Gaussian-distributed although individually they may be. The exact expressions of the joint pdf of the natural frequencies derived in $\S 2$ also support this conclusion for the special cases. Further research is however required to investigate the generality of this conclusion.

\section{Conclusions}

Joint statistics of the natural frequencies of discrete linear dynamic systems with parameter uncertainties have been considered. It is assumed that the mass and stiffness matrices are smooth and at least twice-differentiable functions of a set of random variables. The random variables are in general considered to be non-Gaussian. The usual assumption of small randomness employed in most perturbation methods is not employed in this study. A general theorem on the exact evaluation of the joint probability density function of the natural frequencies of linear stochastic systems has been given. Based on this, some closed-form expressions of the joint probability density function of the natural frequencies have been reported for a few special cases. Due to the lack of generality of the exact methods, an alternative approach based on asymptotic evaluation of multi-dimensional integrals has been suggested. A closedform asymptotically correct expression for general order joint moments of arbitrary number of natural frequencies of linear stochastic systems with general non-Gaussian distribution has been derived for the first time. Using this expression, the joint probability density function of the natural frequencies are calculated and compared with perturbation approximations and Monte Carlo simulation for a three-degree-of-freedom random system. It was observed that the natural frequencies are not jointly Gaussian-distributed although their marginal distributions are Gaussian. Generality of this result however remains to be verified in future studies.

The author acknowledges the support of the UK Engineering and Physical Sciences Research Council (EPSRC) through the award of an Advanced Research Fellowship. 


\section{List of symbols and abbreviations}

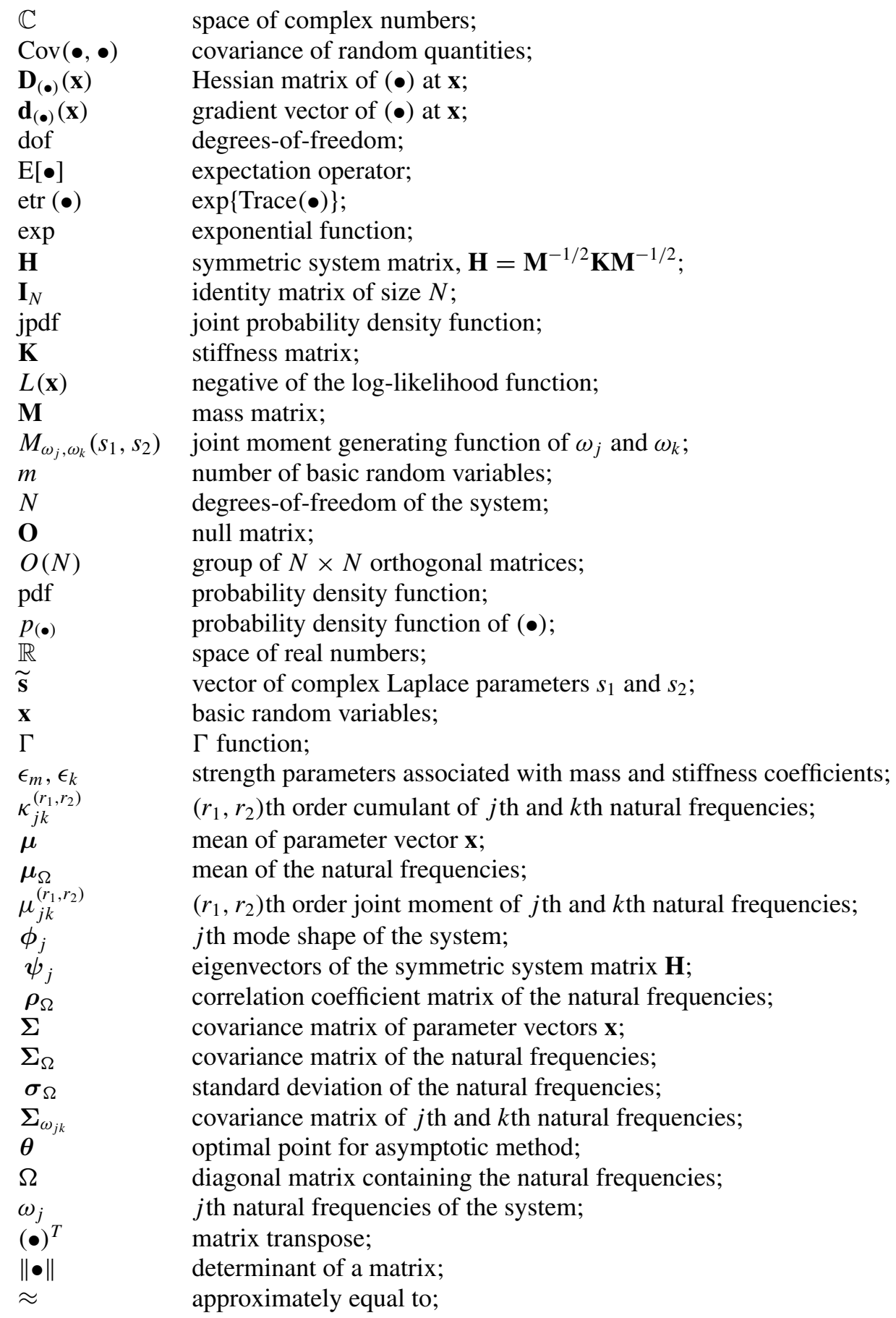




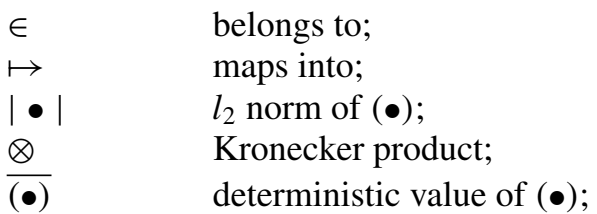

\section{Appendix A. Gradient vector and Hessian matrix of the natural frequencies}

The eigenvectors of symmetric linear systems are orthogonal with respect to the mass and stiffness matrices. Normalize the eigenvectors so that they are unity mass normalized, that is,

$$
\phi_{j}^{T} \mathbf{M} \phi_{j}=1 .
$$

Using this and differentiating (1) with respect to $x_{k}$ it can be shown that (Fox \& Kapoor 1968) for any $\mathbf{x}$

$$
\begin{gathered}
\frac{\partial \omega_{j}(\mathbf{x})}{\partial x_{k}}=\frac{\phi_{j}(\mathbf{x})^{T} \mathcal{G}_{j k}(\mathbf{x}) \phi_{j}(\mathbf{x})}{2 \omega_{j}(\mathbf{x})}, \\
\text { where } \quad \mathcal{G}_{j k}(\mathbf{x})=\left[\frac{\partial \mathbf{K}(\mathbf{x})}{\partial x_{k}}-\omega_{j}^{2}(\mathbf{x}) \frac{\partial \mathbf{M}(\mathbf{x})}{\partial x_{k}}\right] .
\end{gathered}
$$

Differentiating (1) with respect to $x_{k}$ and $x_{l}$, Plaut \& Huseyin (1973) have shown that, providing the natural frequencies are distinct,

$$
\frac{\partial^{2} \omega_{j}(\mathbf{x})}{\partial x_{k} \partial x_{l}}=\left[\frac{1}{2 \omega_{j}(\mathbf{x})} \frac{\partial^{2}\left(\omega_{j}^{2}(\mathbf{x})\right)}{\partial x_{k} \partial x_{l}}-\frac{1}{\omega_{j}(\mathbf{x})} \frac{\partial \omega_{j}(\mathbf{x})}{\partial x_{l}} \frac{\partial \omega_{j}(\mathbf{x})}{\partial x_{k}}\right],
$$

where

$$
\begin{aligned}
\frac{\partial^{2}\left(\omega_{j}^{2}(\mathbf{x})\right)}{\partial x_{k} \partial x_{l}}= & \phi_{j}(\mathbf{x})^{T}\left[\frac{\partial^{2} \mathbf{K}(\mathbf{x})}{\partial x_{k} \partial x_{l}}-\omega_{j}^{2}(\mathbf{x}) \frac{\partial^{2} \mathbf{M}(\mathbf{x})}{\partial x_{k} \partial x_{l}}\right] \phi_{j}(\mathbf{x}) \\
& -\left(\phi_{j}(\mathbf{x})^{T} \frac{\partial \mathbf{M}(\mathbf{x})}{\partial x_{k}} \phi_{j}(\mathbf{x})\right)\left(\phi_{j}(\mathbf{x})^{T} \mathcal{G}_{j l}(\mathbf{x}) \phi_{j}(\mathbf{x})\right) \\
& -\left(\phi_{j}(\mathbf{x})^{T} \frac{\partial \mathbf{M}(\mathbf{x})}{\partial x_{l}} \phi_{j}(\mathbf{x})\right)\left(\phi_{j}(\mathbf{x})^{T} \mathcal{G}_{j k}(\mathbf{x}) \phi_{j}(\mathbf{x})\right) \\
& +2 \sum_{r=1}^{N} \frac{\left(\phi_{r}(\mathbf{x})^{T} \mathcal{G}_{j k}(\mathbf{x}) \phi_{j}(\mathbf{x})\right)\left(\phi_{r}(\mathbf{x})^{T} \mathcal{G}_{j l}(\mathbf{x}) \phi_{j}(\mathbf{x})\right)}{\omega_{j}^{2}(\mathbf{x})-\omega_{r}^{2}(\mathbf{x})} .
\end{aligned}
$$

Equations (A2) and (A4) completely define the elements of the gradient vector and Hessian matrix of the natural frequencies. 


\section{Appendix B. Derivative of the system matrices with respect to the random variables}

The derivatives of $\mathbf{M}(\mathbf{x})$ and $\mathbf{K}(\mathbf{x})$ with respect to elements of $\mathbf{x}$ can be obtained from (66) together with (67) and (68). For the mass matrix we have,

$$
\frac{\partial \mathbf{M}}{\partial x_{1}}=\left[\begin{array}{ccc}
\bar{m}_{1} \epsilon_{m} & 0 & 0 \\
0 & 0 & 0 \\
0 & 0 & 0
\end{array}\right], \quad \frac{\partial \mathbf{M}}{\partial x_{2}}=\left[\begin{array}{ccc}
0 & 0 & 0 \\
0 & \bar{m}_{2} \epsilon_{m} & 0 \\
0 & 0 & 0
\end{array}\right], \quad \frac{\partial \mathbf{M}}{\partial x_{3}}=\left[\begin{array}{ccc}
0 & 0 & 0 \\
0 & 0 & 0 \\
0 & 0 & \bar{m}_{3} \epsilon_{m}
\end{array}\right] .
$$

All other $\partial \mathbf{M} / \partial x_{i}$ are null matrices. For the derivative of the stiffness matrix

$$
\begin{aligned}
& \frac{\partial \mathbf{K}}{\partial x_{4}}=\left[\begin{array}{ccc}
\bar{k}_{1} \epsilon_{k} & 0 & 0 \\
0 & 0 & 0 \\
0 & 0 & 0
\end{array}\right], \quad \frac{\partial \mathbf{K}}{\partial x_{5}}=\left[\begin{array}{ccc}
0 & 0 & 0 \\
0 & \bar{k}_{2} \epsilon_{k} & 0 \\
0 & 0 & 0
\end{array}\right], \quad \frac{\partial \mathbf{M}}{\partial x_{6}}=\left[\begin{array}{lll}
0 & 0 & 0 \\
0 & 0 & 0 \\
0 & 0 & \bar{k}_{3} \epsilon_{k}
\end{array}\right], \\
& \frac{\partial \mathbf{K}}{\partial x_{7}}=\left[\begin{array}{ccc}
\bar{k}_{4} \epsilon_{k} & -\bar{k}_{4} \epsilon_{k} & 0 \\
-\bar{k}_{4} \epsilon_{k} & \bar{k}_{4} \epsilon_{k} & 0 \\
0 & 0 & 0
\end{array}\right], \quad \frac{\partial \mathbf{K}}{\partial x_{8}}=\left[\begin{array}{ccc}
0 & 0 & 0 \\
0 & \bar{k}_{5} \epsilon_{k} & -\bar{k}_{5} \epsilon_{k} \\
0 & -\bar{k}_{5} \epsilon_{k} & \bar{k}_{5} \epsilon_{k}
\end{array}\right], \\
& \frac{\partial \mathbf{M}}{\partial x_{9}}=\left[\begin{array}{ccc}
\bar{k}_{6} \epsilon_{k} & 0 & -\bar{k}_{6} \epsilon_{k} \\
0 & 0 & 0 \\
-\bar{k}_{6} \epsilon_{k} & 0 & \bar{k}_{6} \epsilon_{k}
\end{array}\right],
\end{aligned}
$$

and all other $\partial \mathbf{K} / \partial x_{i}$ are null matrices. Also note that all the first-order derivative matrices are independent of $\mathbf{x}$. For this reason, all the higher order derivatives of the $\mathbf{M}(\mathbf{x})$ and $\mathbf{K}(\mathbf{x})$ matrices are null matrices.

\section{References}

Adhikari S 2004 Complex modes in stochastic systems. Adv. Vibr. Eng. 3: 1-11

Adhikari S, Friswell M I 2004 Random eigenvalue problems in structural dynamics. In 45th AIAA/ASME/ASCE/AHS/ASC Structures, Structural Dynamics \& Materials Conference, Palm Springs, CA, USA

Adhikari S, Langley R S 2003 Distribution of eigenvalues of linear stochastic systems. In Proc. 9th Int. Conf. on Applications of Statistics and Probability in Civil Engineering (ICASP 9), San Fransisco, CA, USA, vol. 1 of Applications of statistics and probability in civil engineering (eds) A Der-Kiureghian, S Madanat, J M Pestana (Rotterdam: Millpress) pp 201-207

Benaroya H 1992 Random eigenvalues, algebraic methods and structural dynamic models. Appl. Math. Comput. 52: 37-66

Bleistein N, Handelsman R A 1994 Asymptotic expansions of integrals (New York: Holt, Rinehart and Winston)

Boyce W E 1968 Random eigenvalue problems. Probabilistic methods in applied mathematics (New York: Academic Press)

Brody T A, Flores J, French J B, Mello P A, Pandey A, Wong S S M 1981 Random matrix physics: Spectrum and strength fluctuations. Rev. Mod. Phys. 53: 385-480

Collins J D, Thomson W T 1969 The eigenvalue problem for structural systems with statistical properties. AIAA J. 7: 642-648

Dumitriu I, Edelman A 2002 Matrix models for beta ensembles. J. Math. Phys. 43: 5830-5847

Fox R L, Kapoor M P 1968 Rates of change of eigenvalues and eigenvectors. AIAA J. 6: 2426-2429 
Friswell M I 1996 The derivatives of repeated eigenvalues and their associated eigenvectors. ASME J. Vibr. Acoust. 18: 390-397

Grigoriu M 1992 A solution of random eigenvalue problem by crossing theory. J. Sound Vibr. 158: $69-80$

Gupta A, Nagar D 2000 Matrix variate distributions (Monographs \& Surveys in Pure \& Applied Mathematics) (London: Chapman \& Hall/CRC)

Hart G C 1973 Eigenvalue uncertainties in stressed structure. J. Eng. Mech., Am. Soc. Civil Eng. 99: 481-494

Hasselman T K, Hart G C 1972 Modal analysis of random structural system. J. Eng. Mech., Am. Soc. Civil Eng. 98: 561-579

Ibrahim R A 1987 Structural dynamics with parameter uncertainties. Appl. Mech. Rev., ASME 40: 309-328

Iyengar R N, Manohar C S 1989 Probability distribution function of the eigenvalues of random string equation. J. Appl. Math. 56: 202-207

Johnson N L, Kotz S 1970 Distributions in statistics: Continuous univariate distributions - 2. The Houghton Mifflin series in statistics (Boston, MA: Houghton Mifflin)

Langley R S, Brown A W M 2001 Numerical evidence for the Wigner surmise. J. Acoust. Soc. Am. 109: 2443

Langley R S, Brown A W M 2004a The ensemble statistics of the band-averaged energy of a random system. J. Sound Vibr., 275: 847-857

Langley R S, Brown A W M 2004b The ensemble statistics of the energy of a random system subjected to harmonic excitation. J. Sound Vibr. 275: 823-846

Lee C, Singh R 1994 Analysis of discrete vibratory systems with parameter uncertainties, part I: Eigensolution. J. Sound Vibr. 174: 379-394

Manohar C S, Gupta S 2003 Modeling and evaluation of structural reliability: Current status and future directions. In Research reviews in structural engineering, Golden Jubilee Publications of Department of Civil Engineering, Indian Institute of Science, Bangalore (eds) K S Jagadish, R N Iyengar (Hyderabad: Universities Press)

Manohar C S, Ibrahim R A 1999 Progress in structural dynamics with stochastic parameter variations: 1987 to 1998. Appl. Mech. Rev. ASME 52: 177-197

Mathai A M, Provost S B 1992 Quadratic forms in random variables: Theory and applications (New York: Marcel Dekker)

Mehta M L 1991 Random matrices 2nd edn (San Diego, CA: Academic Press)

Muirhead R J 1982 Aspects of multivariate statistical theory (New York: John Wiley and Sons)

Nair P B, Keane A J 2003 An approximate solution scheme for the algebraic random eigenvalue problem. J. Sound Vibr. 260: 45-65

Plaut R H, Huseyin K 1973 Derivative of eigenvalues and eigenvectors in non-self adjoint systems. AIAA J. 11: 250-251

Ramu S A, Ganesan R 1993 A Galerkin finite element technique for stochastic field problems. Comput. Meth. Appl. Mech. Eng. 105: 315-331

Sankar T S, Ramu S A, Ganesan R 1993 Stochastic finite element analysis for high speed rotors. J. Vibr. Accoust., ASME 115: 59-64

Scheidt J V, Purkert W 1983 Random eigenvalue problems (New York: North Holland)

Wong R 2001 Asymptotic approximations of integrals (Philadelphia, PA: Soc. Ind. Appl. Math.) (first published by Academic Press in 1989) 